\title{
Método citológico e histopatológiico no diagnóstico das lesões da conjuntiva: estudo comparativo
}

\author{
Cytologic and histologic methods in the diagnosis of conjunctival lesions: \\ comparativestudy
}

\author{
Carlos Gustavo de Melo G. Lima ${ }^{1}$ \\ João Carlos Bezerra Veloso \\ Ana Daniele Tavares ${ }^{3}$ \\ Patrícia Jung'man ${ }^{4}$ \\ André Araújo de Vasconcelos ${ }^{5}$
}

\begin{tabular}{|l|}
\hline RESUMO \\
\hline Objetivos: Avaliar a eficácia e a confiabilidade do método citológico nas \\
lesões da conjuntiva, comparando-o com o exame histopatológico. Méto- \\
dos: Realizou-se estudo prospectivo em 31 lesões conjuntivais. O material \\
para estudo foi coletado raspando-se as lesões com a ajuda da espátula de \\
Kimura. Posteriormente, procedeu-se à exérese das mesmas que foram \\
encaminhadas para oxamehistopatológico. As lâminas doexame citológico \\
foram coradas pela técnica de Papanicolaou. Resultados: O método \\
citológico mostrou alta especificidade (100\%) e sensibilidade (92,9\%). O \\
valor preditivo positivo foi de 100\% e o valor preditivo negativo foi de \\
94,4\%. Conclusões: O simples método citológico é efetivo e seguro no \\
diagnóstico de lesões tumorais da conjuntiva.
\end{tabular}

Descritores: Neoplasias da conjuntiva/diagnóstico; Neoplasias da conjuntiva/ultrasonografia; Conjuntiva/citologia, Conjuntiva/patologia; Técnicas citológicas; Reprodutibilidade de resultados; Estudo comparativo

\footnotetext{
${ }^{1}$ Fellow de Plástica Ocular da Fundação Altino Ventura (FAV). Recife (PE).

${ }^{2}$ Fellow de Plástica Ocular da FAV. Recife (PE).

${ }^{3}$ Oftalmologista da FAV. Recife (PE).

${ }^{4}$ Patologista do Hospital da Restauração. Recife (PE).

${ }^{5}$ Médico do Setor de Plástica Ocular da FAV. Recife (PE).

Endereço para correspondência: Setor de Plástica Ocular - Departamento de Oftalmologia - Fundação Altino Ventura. Rua da Soledade, 170 - Recife (PE) CEP 50070-040 E-mail: carlosgustav@ hotmail.com

Recebido para publicação em 12.05.2004

Versão revisada recebida em 10.02.2005

Aprovação em 13.06.2005

Nota Editorial: Depois de concluída a análise do artigo sob sigilo editorial e com a anuência das Dras. Clélia Maria Erwenne e Maristela Amaral Palazzi sobre a divulgação de seus nomes como revisoras, agradecemos sua participação neste processo.
}

\section{INTRODUÇÃO}

A conjuntiva é sede de lesões de várias etiologias, que incluem alterações degenerativas, circulatórias, inflamatórias, e do crescimento e desenvolvimento, cujo diagnóstico diferencial, apenas pelo exame clínico pode ser impreciso ${ }^{(1-2)}$. Qualquer um dos vários elementos que compõem a conjuntiva e o tecido subconjuntival poderá dar origem a estas lesões ${ }^{(3)}$.

Os tumores conjuntivais representam um amplo espectro de lesões benignas e malignas, algumas capazes de acarretar não somente a perda da visão como, também, da vida. A abordagem correta destas lesões compreende diagnóstico e tratamento apropriados ${ }^{(3)}$.

A técnica básica para obtenção de material para o diagnóstico de uma lesão conjuntival é a biópsia excisional, que tem dupla função: diagnóstica e terapêutica. Além de prover material para o diagnóstico histopatológico, a excisão é freqüentemente curativa. Nos casos em que a excisão, isoladamente, é insuficiente para evitar a recidiva ou o desenvolvimento de metástases do tumor, terapias adjuvantes como a crioterapia, quimioterapia ou radioterapia podem ser utilizadas ${ }^{(4)}$.

A córnea e a conjuntiva são tecidos de acesso fácil, o que possibilita um detalhado exame clínico, além da utilização de técnicas para obtenção de material para exame morfológico, que possam contribuir para o esclarecimento diagnóstico. É significativa a importância da citologia esfoliativa para a diferenciação de lesões tumorais benignas e malignas, sendo o método citológico de Papanicolaou uma técnica laboratorial estabelecida para a detecção de neoplasias em amostras de tecidos. Este método ainda 
pode ser utilizado na avaliação de tumores oculares externos com relatos sugerindo seu potencial valor ${ }^{(5)}$.

Em oftalmologia, a primeira referência ao uso do método de Papanicolaou surgiu em 1954, quando Lamorde e Timset usaram a citologia na avaliação de tumores limbares e encontraram associações positivas entre os achados citológicos e os exames histológicos. Há evidência de que a técnica de Papanicolaou pode ser empregada na investigação de uma variedade de lesões oculares benignas, lesões neoplásicas originárias de células escamosas, bem como no melanoma maligno da conjuntiva e carcinoma de glândula meibomiana ${ }^{(5)}$.

Foi objetivo do presente estudo avaliar a eficácia e a confiabilidade do método citológico como auxílio diagnóstico em casos de lesões conjuntivais, principalmente no diagnóstico diferencial entre lesões de caráter benigno e as de caráter maligno, por meio de comparação com o exame histopatológico.

\section{MÉTODOS}

Realizou-se estudo prospectivo de 31 lesões conjuntivais observadas no departamento de Plástica Ocular da Fundação Altino Ventura no período de julho de 2001 a janeiro de 2003. Dos 31 pacientes estudados, 17 eram do gênero feminino e 14 do masculino. A média de idade foi de 48,5 anos ( $\mathrm{DP}=20,2)$. Tanto as lesões tumorais como as inflamatórias foram incluídas, sendo excluídas aquelas que haviam recebido tratamento prévio, clínico ou cirúrgico. De acordo com seu aspecto ao exame biomicroscópico, as lesões foram classificadas clinicamente em displásicas ou não e especificados os seus prováveis diagnósticos. Nas lesões de aparência maligna, foi realizada a ultra-sonografia biomicroscópica (UBM). O estudo da lesão pela UBM forneceu-nos dados sobre possíveis infiltrações em profundidade tanto da córnea quanto da esclera, que foram importantes para o planejamento cirúrgico. Nas lesões de aparência maligna que apresentavam extensão ao fundo de saco conjuntival, realizou-se a tomografia computadorizada da órbita para avaliação de uma eventual extensão a essa região.

O exame citológico foi obtido por raspado da superfície das lesões da conjuntiva previamente anestesiada com proparacaína a $0,5 \%$, utilizando-se a espátula de Kimura. Dois esfregaços lineares foram obtidos, sendo as lâminas imersas em tubos com solução fixadora (álcool absoluto e éter - 1:1), por tempo nunca inferior a 30 minutos e nem superior a duas semanas. $\mathrm{O}$ material do exame citológico foi processado pela técnica de Papanicolaou, seguindo os princípios de Masson ${ }^{(6)}$.

Posteriormente, os pacientes foram submetidos à exérese cirúrgica da lesão, com enxerto de membrana amniótica e crioterapia em alguns casos selecionados. Deve-se enfatizar ainda a importância da biópsia de congelação na orientação das margens de ressecção. As peças foram fixadas em formalina a $10 \%$ e enviadas para estudo histopatológico. O material foi processado e corado com o método da hematoxilina e eosina. As lesões foram classificadas em malignas, pré-malignas e benignas de acordo com a histologia e características citológi- cas do epitélio de revestimento. Todos os exames foram realizados pela mesma patologista.

Foram utilizados parâmetros de avaliação dos testes diagnósticos como sensibilidade, especificidade, valor preditivo positivo (VPP) e valor preditivo negativo (VPN), considerando-se o exame histopatológico como o "padrão ouro". Para avaliação da concordância, utilizou-se o teste kappa. Aceitouse um valor de kappa $\geq$ que 0,8 como forte concordância. Aceitou-se a hipótese de nulidade quando p>0,05.

\section{RESULTADOS}

Entre as 8 lesões classificadas como malignas pelo exame histopatológico, 6 foram classificadas da mesma forma (Figura 1) e 2 como lesões pré-cancerosas, pelo método citológico. Das 6 lesões classificadas como pré-cancerosas pelo exame histopatológico, 5 foram classificadas da mesma forma e 1 como lesão benigna pelo método citológico (Figura 2). Todas as lesões classificadas como benignas pelo exame histopatológico, foram classificadas da mesma forma pelo método citológico (Figura 3). Existiu uma forte concordância entre exame

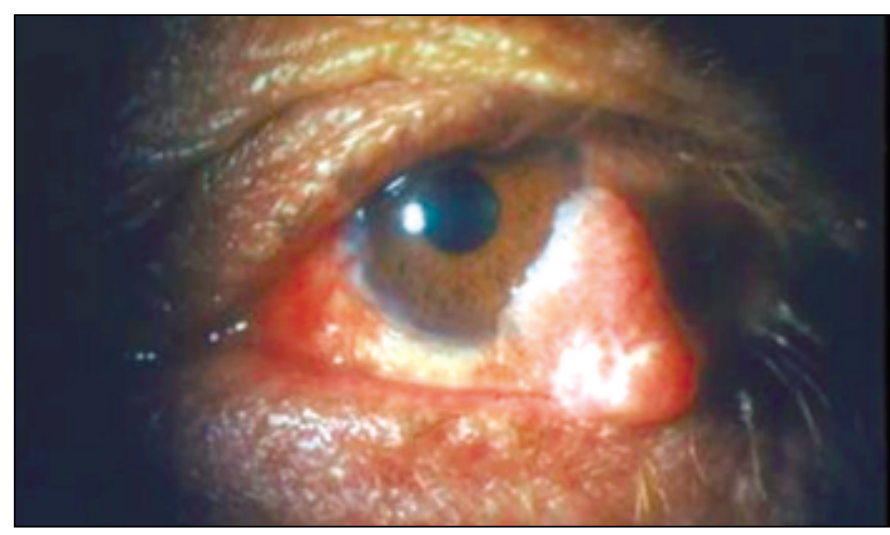

Figura 1 - Carcinoma espinocelular, em região ínfero-lateral do olho esquerdo, diagnosticado pelo método citológico e pela histopatologia

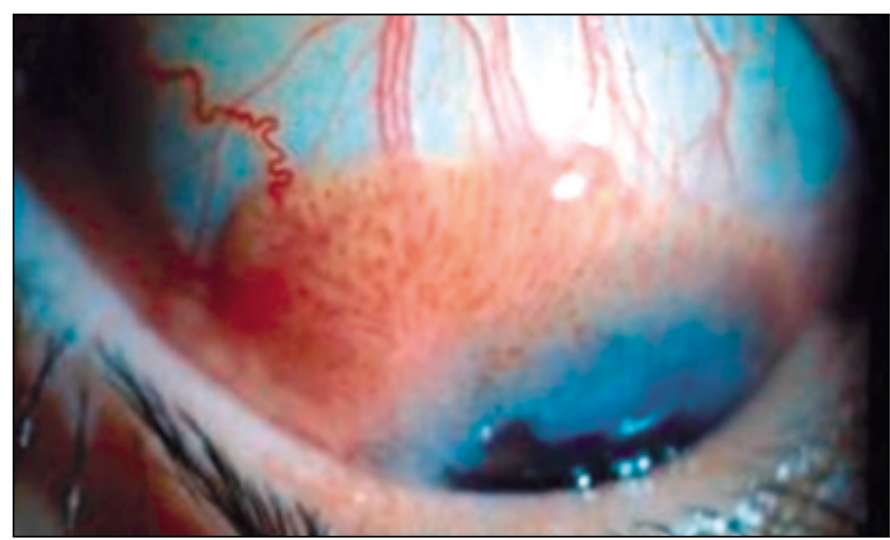

Figura 2 - Lesão mista: porção conjuntival sugestiva de lesão papilomatosa e porção corneana apresentando características de displasia epitelial em região superior do olho direito 
citológico e o histopatológico, sendo o coeficiente de concordância de kappa de 0,835. A distribuição das frequiências dos diagnósticos citológicos e histopatológicos está na tabela 1.

Os diagnósticos biomicroscópicos e citológicos das le-

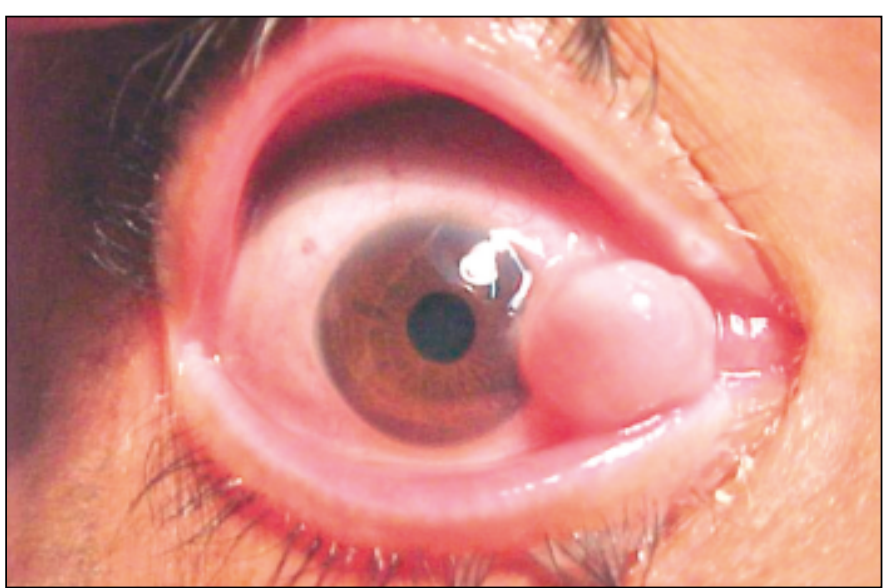

Figura 3 - Granuloma piogênico em canto nasal da conjuntiva bulbar do olho direito, diagnosticado pelo exame citológico e histopatológico sões conjuntivais foram estudados, verificando-se a possível associação com o exame histopatológico. É possível observar que em 2 casos (pacientes 17 e 28), o exame citológico evidenciou o diagnóstico de lesões pré-cancerosas (lesões displásicas), que na verdade se tratavam de lesões malignas (carcinoma espinocelular), pelo exame histopatológico. Pode ser visto ainda que em 1 caso (paciente 13), houve o diagnóstico inicial de hiperplasia pelo exame citológico, quando na verdade se tratava de uma lesão displásica, confirmada pelo exame histopatológico (Tabela 2).

\begin{tabular}{|c|c|c|c|c|}
\hline \multirow[b]{2}{*}{ Citológico } & \multicolumn{3}{|c|}{ Histopatológico } & \multirow[b]{2}{*}{ Total } \\
\hline & Maligna & Pré-maligna & Benigna & \\
\hline Maligna & $6(19 \%)$ & $0(0 \%)$ & $0(0 \%)$ & $6(19 \%)$ \\
\hline Pré-maligna & $2(7 \%)$ & $5(1$ & $0(c$ & 7 ( $23 \%)$ \\
\hline Benigna & $0(0$ & $1 i 3$ & 17 & $18(58 \%)$ \\
\hline Total & 8 (26\%) & $6(19 \%)$ & $17(55 \%)$ & $31(100 \%)$ \\
\hline
\end{tabular}

\begin{tabular}{|c|c|c|c|c|c|}
\hline Caso & Idade & Sexo & Biomicroscopia & Citológico & Histopatológico \\
\hline 1 & 66 & $\mathrm{~F}$ & Pterígio & Pterígio & Pterígo \\
\hline 2 & 39 & $M$ & Pterígio & Pterígio & Pterígo \\
\hline 3 & 21 & $\mathrm{~F}$ & Granuloma & Granuloma & Granuloma piogênico \\
\hline 4 & 21 & M & Granuloma & Granuloma & Granuloma piogênico \\
\hline 5 & 77 & M & Carcinoma espinocelular & Displasia & Displasia \\
\hline 6 & 77 & M & Displasia & Displasia & Displasia \\
\hline 7 & 47 & $\mathrm{~F}$ & Displasia & Carcinoma espinocelular & Carcinoma espinocelular \\
\hline 8 & 70 & $\mathrm{~F}$ & Ceratose actínica & Displasia & Displasia \\
\hline 9 & 31 & M & Displasia & Pterígio & Pterígio \\
\hline 10 & 50 & $\mathrm{~F}$ & Carcinoma espinocelular & Carcinoma espinocelular & Carcinoma espinocelular \\
\hline 11 & 73 & $\mathrm{~F}$ & Carcinoma espinocelular & Displasia & Displasia \\
\hline 12 & 70 & $M$ & Carcinoma espinocelular & Papiloma & Papiloma \\
\hline 13 & 8 & $\mathrm{~F}$ & Displasia & Hiperplasia & Displasia \\
\hline 14 & 47 & M & Papiloma & Papiloma & Papiloma \\
\hline 15 & 54 & $\mathrm{~F}$ & Granuloma & Granuloma & Granuloma piogênico \\
\hline 16 & 50 & $M$ & Pinguécula & Pinguécula & Pinguécula \\
\hline 17 & 24 & $\mathrm{~F}$ & Displasia & Displasia & Carcinoma espinocelular \\
\hline 18 & 56 & M & Granuloma & Granuloma & Granuloma piogênico \\
\hline 19 & 43 & M & Pterígio & Pterígio & Pterígio \\
\hline 20 & 16 & $\mathrm{~F}$ & Pterígio & Pterígio & Pterígio \\
\hline 21 & 79 & $M$ & Carcinoma espinocelular & Carcinoma mucoepidermóide & Carcinoma mucoepidermóide \\
\hline 22 & 28 & $\mathrm{~F}$ & Nevo & Nevo & Nevo \\
\hline 23 & 30 & $\mathrm{~F}$ & Leucoplasia & Leucoplasia & Leucoplasia \\
\hline 24 & 50 & M & Displasia & Carcinoma espinocelular & Carcinoma espinocelular \\
\hline 25 & 56 & $\mathrm{~F}$ & Pterígio & Pterígio & Pterígio \\
\hline 26 & 69 & M & Carcinoma espinocelular & Carcinoma espinocelular & Carcinoma espinocelular \\
\hline 27 & 29 & $\mathrm{~F}$ & Papiloma & Papiloma & Papiloma \\
\hline 28 & 71 & $\mathrm{~F}$ & Carcinoma espinocelular & Displasia & Carcinoma espinocelular \\
\hline 29 & 35 & $\mathrm{~F}$ & Pinguécula & Pinguécula & Pinguécula \\
\hline 30 & 59 & $\mathrm{~F}$ & Papiloma & Displasia & Displasia \\
\hline 31 & 58 & M & Carcinoma espinocelular & Carcinoma espinocelular & Carcinoma espinocelular \\
\hline
\end{tabular}


O cálculo dos parâmetros de sensibilidade, especificidade, valor preditivo positivo (VPP) e valor preditivo negativo (VPN), foi realizado segundo a distribuição dos resultados. Obtiveram-se os seguintes resultados: sensibilidade $(92,9 \%)$; especificidade (100\%); VPP (100\%); VPN (94,4\%) (Tabela 3).

\section{DISCUSS ÃO}

A citologia do tecido de descamação da conjuntiva e córnea permite o exame das camadas celulares superficiais. Essas células são derivadas das camadas de células basais ou germinativas, as quais sofrem alterações morfológicas no seu processo de maturação. A maturação anormal das células neoplásicas irá, portanto, ser refletida em células superficiais com características anômalas. Esse princípio da citologia permite o estudo do tecido através do exame da morfologia das células da superfície ${ }^{(5)}$.

Neste estudo, foi possível observar a discordância entre os métodos citológico e histopatológico em três casos $(13,17$, 28 ), como pode ser observado na tabela 1 . Estes resultados podem ser atribuídos a alguns fatores, como: erro de avaliação do tipo citológico encontrado; dificuldade de obtenção do material, devido à menor descamação do epitélio conjuntival, quando comparado ao da cérvice uterina, o que pode fazer com que determinada amostra não represente integralmente a lesão; pode-se citar ainda as dificuldades na fixação e na coloração do esfregaço ${ }^{(4)}$. A região onde foi realizado o raspado também pode ser responsável por erros na interpretação diagnóstica, já que é freqüente a presença de displasia na margem corneana de um carcinoma espinocelular. No caso 13, por exemplo, foi possível evidenciar tanto pelo exame biomicroscópico, como pela histopatologia, que se tratava de uma lesão claramente mista: porção conjuntival sugestiva de lesão papilomatosa e porção corneana apresentando características de displasia epitelial. No entanto, o resultado da citologia evidenciou uma lesão benigna (hiperplasia epitelial). É possível que neste caso especificamente o local onde foi realizado o raspado tenha influenciado diretamente no resultado falsonegativo (Figura 2). Esse problema pode ser resolvido através da realização de um maior número de colheitas, o que levaria a um aumento na porcentagem de acertos ${ }^{(4)}$. Em casos de dúvida diagnóstica, a citologia permite ainda um novo estudo, na mesma lesão, sem que ocorram alterações decorrentes do primeiro procedimento ${ }^{(4-5)}$.

Os resultados encontrados neste trabalho foram semelhantes aos descritos na literatura. Demonstrou-se, com o método, alta especificidade para o diagnóstico de lesões conjuntivais, além de ausência de resultados falso-positivos, já que todos os diagnósticos citológicos de malignidade foram confirmados pelo exame histológico ${ }^{(4)}$. A neoplasia maligna mais freqüente da conjuntiva foi o carcinoma espinocelular, concordando com o resultado de outros estudos ${ }^{(2,7)}$.

\begin{tabular}{|lcc|}
\hline $\begin{array}{l}\text { Tabela } \\
\text { 3. Sensibilidade, especificidade, valor preditivo positivo e } \\
\text { valor preditivo negativo para o método citológico }\end{array}$ \\
& $\%$ & IC $95 \%^{*}$ \\
Sensibilidade & 92,9 & $68,5-98,7$ \\
Especificidade & 100,0 & $81,6-100,0$ \\
Valor preditivo positivo (VPP) & 100,0 & $77,2-100,0$ \\
Valor preditivo negativo (VPN) & 94,4 & $74,2-99,0$ \\
${ }^{*} \mathrm{IC}=$ intervalo de confiança & & \\
\hline
\end{tabular}

\section{CONCLUSÕES}

O exame citológico da conjuntiva se mostrou um procedimento simples, que fornece informações valiosas no estudo de lesões oculares externas. Nesse sentido, o método pode ser considerado eficaz, confiável e de fácil execução.

\section{ABSTRACT}

Purpose: To evaluate both the efficacy and reliability of the cytologic method in the diagnosis of conjunctival lesions and compare it with the histologic examination. Methods: A prospective study on 31 conjunctival lesions was performed. Samples were obtained with a Kimura spatula. The lesions were, then, surgically removed and sent for histologic examination. The Papanicolaou technique was used for the cytologic examination. Results: The cytologic method showed high specificity (100\%) and sensitivity (92.9\%). The positive predictive value was $100 \%$ and the negative was $94.4 \%$. Conclusions: The simple cytologic method is effective and reliable for the diagnosis of tumoral lesions of the conjunctiva.

Keywords: Conjunctival neoplasms/diagnosis; Conjunctival neoplasms/ultrasonography; Conjunctiva/cytology; Conjunctiva/pathology; Cytological techniques, Reproducibility of results; Comparative study

\section{REFERÊNCIAS}

1. Neves RA, Pavésio CEN, Nosé W, Belfort Júnior R. Coloração in vivo para o diagnóstico de lesões neoplásicas e displásicas da conjuntiva. Arq Bras Oftalmol. 1993;56(6):308-14.

2. Kato ET, Macruz E, Morais L, Sabrosa NA, Holzchuh N, Alves MR, et al. Tumores conjuntivais: ocorrência na clínica oftalmológica da Faculdade de Ciências Médicas da UNICAMP. Rev Bras Oftalmol. 1996;55(12):921-5.

3. Santo RM. Tumores conjuntivais. In: Alves MR. Conjuntiva cirúrgica. São Paulo: Roca; 1999. p.125-7.

4. Pavésio CE, Burnier Júnior M, Belfort Júnior R. Avaliação do método citológico no diagnóstico de lesões tumorais da conjuntiva. Arq Bras Oftalmol. 1994;57(2):80-3.

5. Gelender H, Forster RK. Papanicolaou cytology in the diagnosis and management of external ocular tumors. Arch Ophthalmol. 1980;98(5):909-12.

6. Michalany J. Técnica histológica em anatomia patológica: com instruções para o cirurgião, enfermeira e citotécnico. São Paulo: EPU; 1981. p.182-6.

7. Burnier Júnior M, Belfort Júnior R, Rigueiro MP, Montezzo LC, Chiferi Júnior V. Neoplasias malignas da conjuntiva. Arq Inst Penido Burnier. 1988;30(2):80-3. 